\title{
PHOTODISSOCIATION REGIONS AND PLANETARY NEBULAE
}

\author{
A.G.G.M. TIELENS \\ MS 245-3, NASA Ames Research Center, Moffett Field, CA 94035-1000, USA
}

\begin{abstract}
FUV photons $(<13.6 \mathrm{eV})$ from the central star create a region of warm $(\approx 1000 \mathrm{~K})$ atomic and molecular gas around Planetary Nebulae $(\mathrm{PN})$. This paper reviews theoretical and observational characteristics of such regions, commonly called photodissociation regions or PDRs. PDRs around PN differ in some aspects from those in other galactic objects and this is briefly discussed with an emphasis on time dependent effects. It is concluded that, in evolved $\mathrm{PN}\left(\mathrm{t}_{\exp }>10^{3} \mathrm{yr}\right)$, molecules will only survive inside dense clumps $\left(>10^{6} \mathrm{~cm}^{-3}\right) . \mathrm{H}_{2}$ emission from such dense gas will show a thermal spectrum in the low v states. Finally, the physical conditions in the PDR associated with NGC 7027 are compared to those in other galactic and extragalactic PDRs
\end{abstract}

\section{Introduction}

Low mass stars lose a considerable fraction of their mass in the form of a dusty molecular wind on the asymptotic giant branch, possibly starting as a gentle breeze $\left(\dot{M} \approx 10^{-6} \mathrm{M}_{\odot} / \mathrm{yr}\right.$; $\mathrm{v}=10 \mathrm{~km} / \mathrm{s})$ which later on turns into a superwind $\left(\dot{\mathrm{M}} \approx 10^{-4} \mathrm{M}_{\odot} / \mathrm{yr} ; \mathrm{v}=15 \mathrm{~km} / \mathrm{s}\right)$. During the rapid evolution of the central star in the transition phase and the early parts of the planetary nebula stage, increasingly higher energy UV photons will illuminate this circumstellar shell and carve out an ionized and an atomic zone in the molecular gas (Pottasch 1980). The ionized gas gives rise to the visibly dominant planetary nebulae itself while the atomic and molecular gas region, commonly called a PhotoDissociation Region (PDR), only lights up in the infrared and submillimeter through atomic finestructure lines, molecular rotational/vibrational lines and the dust continuum.

Over the last two decades, numerous infrared and submillimeter studies have been made of the characteristics of AGB outflows (cf., Habing elsewhere in this volume). By comparison, observations of atomic and molecular gas surrounding planetary nebulae are more limited (cf., Huggins elsewhere in this volume). Molecular observations have largely been limited to the CO 1-0 and 2-1 lines and the $\mathrm{H}_{2}$ 1-0 S(1) line (Huggins and Healy 1989; Zuckerman and Gatley 1988). The predominantly neutral atomic gas in the PDR has been observed in the $21 \mathrm{~cm} \mathrm{HI}$ line (Taylor et al. 1990) and the [CII] $158 \mu \mathrm{m}$ and the [OI] 
63 and $146 \mu \mathrm{m}$ finestructure lines (Dinerstein 1991). These observations reveal that the mass of atomic and molecular material surrounding planetary nebulae can be substantial. For example, for the young compact PN NGC 7027, a conservative lower limit, based upon optically thin $\mathrm{CO}$ emission, places the molecular gas mass at $\approx 0.2 \mathrm{M}_{\odot}$ (Huggins and Healy 1989), including various realistic correction factors would increase this to $\approx 1.4 \mathrm{M}_{\odot}$ (Jaminet et al. 1991). Submillimeter dust continuum emission observations also yield $\approx 1 \mathrm{M}_{\odot}$ (Sopka et al. 1985). In contrast, the ionized gas mass is only $\approx 0.08 \mathrm{M}_{\odot}$ comparable to the atomic gas associated with the CII zone $\left(\approx 0.1 \mathrm{M}_{\odot}\right)$.

Clearly, atomic and molecular gas is an important component of PNe and further studies of their properties are crucial for a proper understanding of the dynamical and morphological evolution of PNe. This paper reviews our theoretical and observational understanding of PDRs. Since little work has been done on PDRs surrounding PNe, the emphasis will be on PDRs associated with HII regions and reflection nebulae ( $\$ 2)$. In $\S 3$ some of the differences of PDRs in PNe will be pointed out. The origin of the observed $\mathrm{H}_{2}$ emission - shocks versus fluorescence - will be assessed in $\$ 4$. Physical conditions in the NGC 7027 PDR are contrasted in $\$ 5$ with those in galactic and extragalactic PDRs. Finally, in $\S 6$, the importance of clumps in PNe is reemphasized.

\section{PDR Models}

Photodissociation regions (PDRs) are regions where FUV $(<13.6 \mathrm{eV})$ photons dominate the heating and/or chemical composition of the gas. PDRs are associated with HII regions, reflection nebulae, bright rim clouds, galactic nuclei, AGNs, and of course PNe. Indeed, penetrating FUV photons from the average interstellar radiation field dominate the chemical composition and heating of molecular clouds for $A_{V}<4$. Thus, besides HI, most of the molecular mass is in PDRs as well. Detailed theoretical models have been developed for the interaction of FUV photons with atomic and molecular gas and dust (Tielens and Hollenbach 1985a hereafter TH; Wolfire et al. 1989; Sternberg and Dalgarno 1989; Burton et al. 1990; Hollenbach et al. 1991). Figure 1 shows the results of a detailed calculation for the physical structure of the PDR in Orion (density $n_{0}=2.3 \times 10^{5} \mathrm{~cm}^{-3}$; incident UV field $\mathrm{G}_{0}=10^{5}$ times the average interstellar radiation field, $1.6 \times 10^{-3} \mathrm{erg} \mathrm{cm}^{-2} \mathrm{~s}^{-1}$; Habing 1968). Basically, the penetrating FUV photons dissociate and ionize the molecular gas to neutral and singly ionized atoms (ie., $\mathrm{H}^{\circ}, \mathrm{C}^{+}, \mathrm{O}^{\circ}, \mathrm{Si}^{+}, \ldots$ ). Deeper in, due to dust attenuation $\left(A_{v}=2\right)$, hydrogen transforms into $\mathrm{H}_{2}$. Similarly, around $\mathrm{A}_{v}=4$, the carbon balance shifts from $\mathrm{C}^{+}$to $\mathrm{C}^{0}$ and $\mathrm{CO}$ (Fig. 1). Except for the fraction locked up in $\mathrm{CO}$, most of the oxygen stays in atomic form until very deep in the cloud $\left(A_{v}>10\right)$ ion-molecule chemistry produces $\mathrm{O}_{2}$. Besides dominating the chemical balance, $\mathrm{FUV}$ photons also control the energy balance. FUV photons can ionize dust grains and PAH molecules and the excess kinetic energy carried away by the photoelectrons will heat the gas. Typically, about $0.5 \%$ of the flux absorbed by the dust is converted into gas heating this way. The remainder $(\approx 99.5 \%)$ heats the dust and is reradiated as IR continuum. Even when the UV is attenuated $\left(A_{\vee}>4\right)$, penetrating red and infrared photons still keep the dust warm and gasgrain collisions dominate the gas heating. The gas cools through the emission of atomic fine structure lines (ie., [OI] $63 \mu \mathrm{m} \&[\mathrm{CII}] 158 \mu \mathrm{m})$ at the surface $(\mathrm{Av}<4 \mathrm{mag})$ and molecular rotational lines (ie., $\mathrm{CO}$ ) deeper in (Fig. 1). At the surface, the gas is generally much hotter than the dust while deeper in the gas temperature drops to somewhat below the 

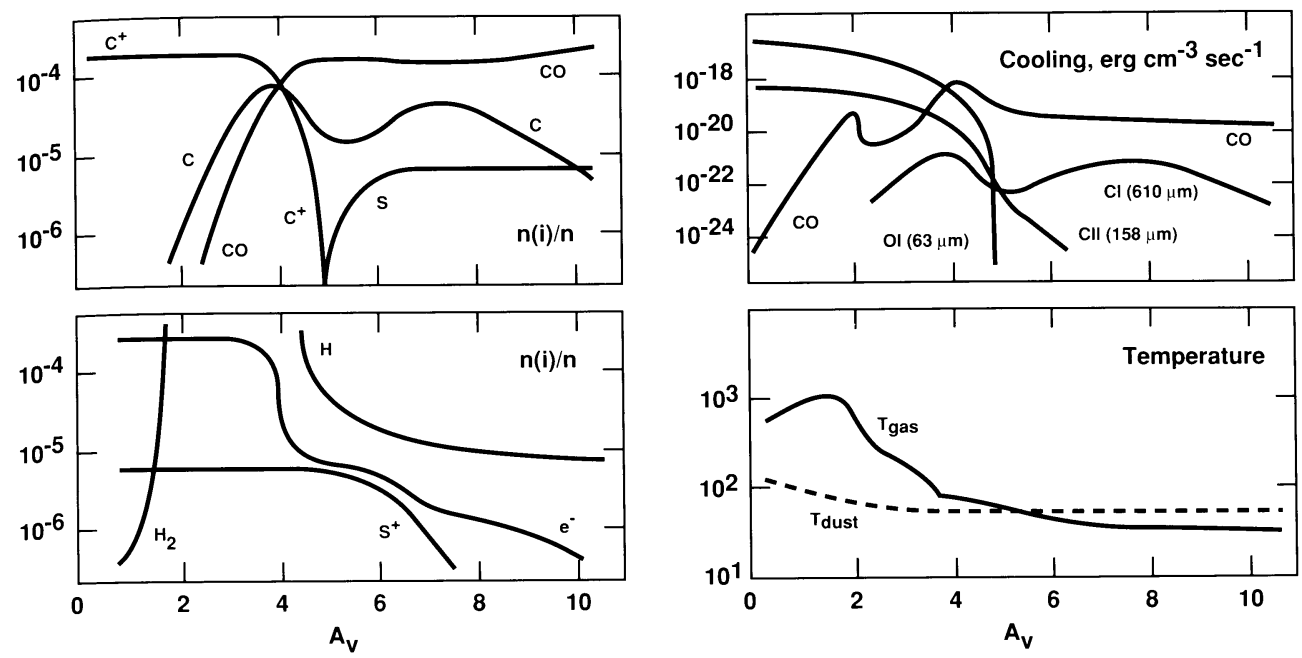

Figure 1: Structure of the PDR in Orion $\left(\mathrm{n}_{\mathrm{o}}=2.3 \times 10^{5} \mathrm{~cm}^{-3}, \mathrm{G}_{\mathrm{o}}=10^{5}\right.$; Tielens and Hollenbach 1985). Left: Abundances relative to total hydrogen as a function of visual extinction into the PDR. Right: Cooling in various lines and gas and dust temperature in the PDR.

dust temperature (Fig. 1).

The chemical structure of a PDR is largely dependent on the penetration of the UV photons and thus on the dust extinction. For species whose photodissociation is dominated by line absorption, self-shielding can be important as well. However, for bright PDRs, this requires high densities. Thus, when $\mathrm{n}_{\mathrm{O}} / \mathrm{G}_{\mathrm{O}}>50 \mathrm{~cm}^{-3}, \mathrm{H}_{2}$ self-shielding becomes important and the $\mathrm{Ho} / \mathrm{H}_{2}$ transition is pulled to the cloud surface. Typically, hydrogen goes then molecular around $A_{v} \approx 0.3$. The upwardly revised $\mathrm{CO}$ photodissociation rates make $\mathrm{CO}$ selfshielding of little importance in PDRs (van Dishoeck and Black 1988). However, the increased $\mathrm{H}_{2}$ abundance near the surface when $\mathrm{H}_{2}$ self shielding is important leads to rapid $\mathrm{CO}$ formation and as a result appreciable amounts of $\mathrm{CO}$ are present near the surface as well (Burton et al. 1990). Further at these high densities and temperatures, collisional deexcitation of UV-pumped vibrationally excited $\mathrm{H}_{2}$ is an important heating source for the gas. High density, bright PDRs have therefore a thin $(A v \approx 0.03)$ very warm $(T \approx 2000 K)$, partly molecular, surface layer.

In summary, PDR models predict a large column density $-N_{O} \approx 8 \times 10^{21} \mathrm{~cm}^{-2}\left(A_{V} \approx 4\right)$ - of warm, atomic gas $(\approx 500 \mathrm{~K})$ at the surface and a somewhat cooler $(\approx 50 \mathrm{~K})$ column of molecular gas. The PDR will be bright in the far-IR dust continuum and PAH emission features at 3.3, 6.2, 7.7, and $11.3 \mu \mathrm{m}$. Particularly, these near-IR emission features traces the penetration of the UV photons. The atomic surface layer $\left(A_{V}<4\right.$ mag) will emit copiously in the $[\mathrm{OI}],[\mathrm{CII}]$, [SiII], and [CI] atomic finestructure lines. The fluorescing near-IR rovibrational lines of $\mathrm{H}_{2}$ will originate around $\mathrm{A}_{\mathrm{v}}=2 \mathrm{mag}$. Rotational $\mathrm{CO}$ emission arises from the cooler molecular gas deeper in $\left(A_{v}>4\right.$ mag). Finally, the very warm surface layer in high density PDRs will be bright in high level CO (ie., 17-16, 14-13) and, reflecting the importance of collisional deexcitation, $\mathrm{H}_{2}$ emission in the low $\mathrm{v}$ states from this gas will show a thermal near-IR spectrum. High $v$ states will still be fluorescing. 


\section{PDRs in Planetary Nebulae}

The physics and chemistry of PDRs in PNe is in some aspects different from that in most other regions. First, in contrast to other regions, PNe may show strong time dependent effects associated with the evolution of the central star (ie., increase in $\mathrm{T}_{\text {eff }}$ ). Second, the dynamical evolution of the nebula may result in weak shocks driven into the atomic and molecular gas. The expected shock velocities range from $5-15 \mathrm{~km} / \mathrm{s}$ during the ionization phase to almost $30 \mathrm{~km} / \mathrm{s}$ in the nebular expansion phase (Marten and Schönberner 1991). Shocks driven into high density clumps will be much weaker. Third, the elemental abundance ratio can be quite different from that in galactic sources (ie., N/O, C/O). Fourth, planetary nuclei may emit appreciable fraction of their energy in the form of soft X-rays, which can penetrate through the ionized volume and heat and ionize the atomic and molecular gas. In that respect PDRs in PNe may resemble those around AGNs. Finally, cosmic rays, which play a major role in interstellar chemistry, are tied to the galactic magnetic field. With a typical gyration radius of $10^{11} \mathrm{~cm}$, cosmic rays do not penetrate into the bubble blown by the red giant and thus they play no role in the chemistry of AGB or $\mathrm{PN}$ ejecta. Space does not permit a thorough discussion of all of these effects and here we will concentrate on the time dependent effects.

First, consider $\mathrm{H}_{2}$ reformation: $\mathrm{H}$ atoms will chemically adsorb on graphitic and silicate surfaces with binding energies of $\approx 2 \mathrm{eV}$ (Tielens and Allamandola 1987). Even at dust temperatures of $1000 \mathrm{~K}$, the grain surface will be completely covered by chemisorbed $\mathrm{H}$. Since dust temperatures in PNe are generally in excess of $50 \mathrm{~K}$, physisorbed $\mathrm{H}$ will evaporate before reacting with another $\mathrm{H}$. $\mathrm{H}_{2}$ formation occurs then through direct reaction of impinging $\mathrm{H}$ atoms with chemisorbed $\mathrm{H}$. Taking the activation barrier of this reaction into account, the efficiency of this reaction is $\varepsilon \approx 0.3 \exp (-1000 / \mathrm{T})$ of the $\mathrm{H}$-collision rate, with $\mathrm{T}$ the gas temperature (Tielens and Allamandola 1987). For $\mathrm{T}=500 \mathrm{~K}$, appropriate for a $\mathrm{PDR}, \varepsilon \approx 0.04$. The timescale to reach $\mathrm{H} / \mathrm{H}_{2}$ equilibrium is then $\approx 7 \times 10^{9} / \mathrm{n}_{\mathrm{O}} \mathrm{yr}$ (Hill and Hollenbach 1978). Clearly, $\mathrm{H}_{2}$ will only reform inside dense clumps $\left(>10^{6} \mathrm{~cm}^{-3}\right)$.

Now, consider $\mathrm{H}_{2}$ photodissociation: The photodissociation timescale is a strong function of the effective temperature of the star. Spaans et al. (1992) have calculated photodissociation rates for constant luminosity $\left(6000 \mathrm{~L}_{\odot}\right)$ star at a distance of $5 \times 10^{16} \mathrm{~cm}$ ( $\mathrm{t}_{\mathrm{exp}}=10^{3} \mathrm{yr}$ ), corresponding to $\mathrm{G}_{\mathrm{o}}=5 \times 10^{5}$ (Fig. 2). For a $6000 \mathrm{~K}$ star, the $\mathrm{H}_{2}$ photodissociation lifetime, about $10^{3}$ times that in the average ISM, is much longer than the expansion timescale. However, for stars earlier than $\mathrm{A} 5, \mathrm{H}_{2}$ photodissociation becomes important and for a typical young $\mathrm{PN}$ with $\mathrm{T}_{\text {eff }}>25000 \mathrm{~K}$ an $\mathrm{H}_{2}$ molecule survives less than a year. Thus, at that point, an $\mathrm{H}_{2}$ dissociation wave will propagate rapidly into the molecular gas. This wave will stop when dust shielding has increased the $\mathrm{H}_{2}$ lifetime (ie., $\left.A_{v} \approx 4\right)$. However, for a typical spherically expanding superwind, the circumstellar dust shell becomes transparent after only $600 \mathrm{yr}$. Alternatively, in clumps, $\mathrm{H}_{2}$ self shielding could be important if $\mathrm{H}_{2}$ reforms rapidly $\left(\mathrm{n}_{\mathrm{O}}>10^{6} \mathrm{~cm}^{-3}\right)$ and this will produce a regular PDR with an $\mathrm{H}^{\circ}-\mathrm{H}_{2}$ transition around $\mathrm{A}_{\mathrm{v}} \approx 0.3-2$, depending on $\mathrm{n}_{\mathrm{O}} / \mathrm{G}_{\mathrm{O}}(\S 2)$. In conclusion, in evolved $\mathrm{PNe}\left(\mathrm{t}_{\exp }>10^{3} \mathrm{yr}\right), \mathrm{H}_{2}$ will only be present inside dense clumps.

A similar discussion can be held for $\mathrm{CO}$. Various channels contribute to $\mathrm{CO}$ reformation (ie., $\mathrm{O}+\mathrm{CH}, \mathrm{CH}_{2}, \mathrm{CH}_{3}{ }^{+}$). The reformation timescale is governed by the radiative association of $\mathrm{C}^{+}$and $\mathrm{H}_{2}$ and is $\approx 10^{8} / \mathrm{n}_{\mathrm{O}} \mathrm{yr}$. However, as these chemical routes necessitate $\mathrm{H}_{2}$, efficient $\mathrm{CO}$ reformation also occurs only inside dense clumps. As for $\mathrm{H}_{2}$, $\mathrm{CO}$ will survive photodestruction through the transition stage to the PN phase (Fig. 2). $\mathrm{CO}$ 


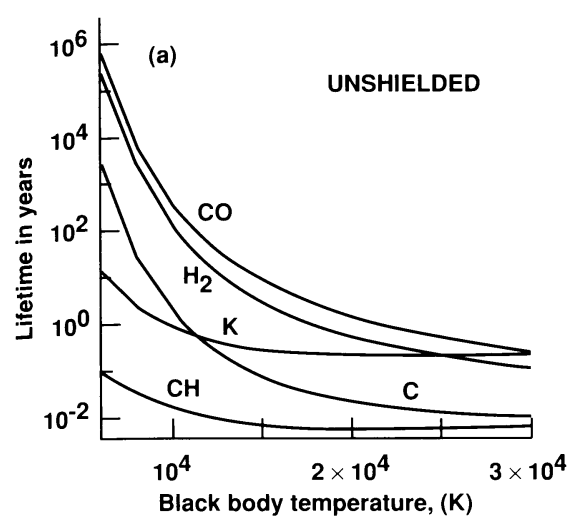

Figure 2: Photo-ionization and dissociation timescales as a function of the effective temperature of the stellar radiation field (Spaans et al. 1992).

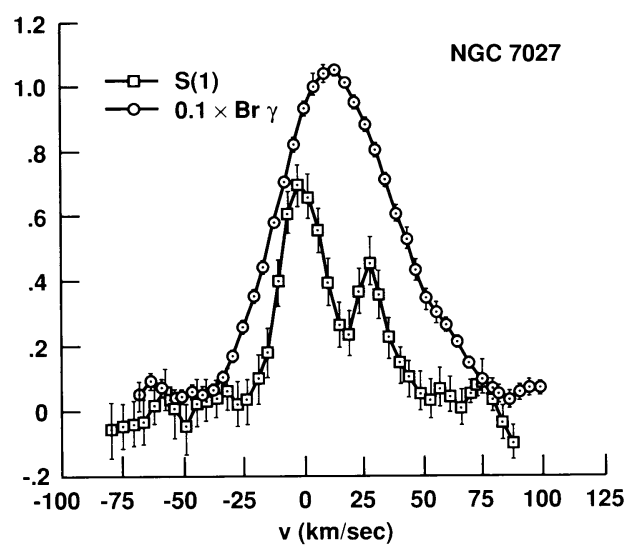

Figure 3: The observed profile of the $\mathrm{H}_{2}$ 1-0 S(1) line compared to the Bry line in NGC 7027 (Geballe 1990).

self shielding is generally of little importance in PDRs but, in the PN phase, dust will raise the lifetime to longer than $10^{3} \mathrm{yr}$ for $\mathrm{A}_{\mathrm{v}}>2.5$. Thus, $\mathrm{CO}$ ejected by the red giant can survive into the PNe phase as long as the clump/envelope is thick enough. However, as for $\mathrm{H}_{2}$, in evolved $\mathrm{PNe}, \mathrm{CO}$ will only be present in high density clumps.

Many molecules can be dissociated by rather mild UV photons compared to $\mathrm{CO}$ and $\mathrm{H}_{2}$ and as a result have short lifetimes even for a $6000 \mathrm{~K}$ star (Figure 2). Again, in the transition phase, such molecules in the AGB superwind can survive due to dust shielding $\left(A_{v}>4\right.$ or more). Their presence in evolved PNe, however, implies high density clumps. In such dense clumps an active chemistry will take place (Cox et al. 1992). HCN formation, for example, might be initiated through reactions of $\mathrm{N}$ with various small hydrocarbon radicals $\left(\mathrm{CH}, \mathrm{CH}_{2}, \mathrm{CH}_{3}{ }^{+}\right)$. Likewise, $\mathrm{HCO}^{+}$might result from reactions of $\mathrm{O}$ with these same species.

\section{$4 \quad \mathrm{H}_{2}$ emission: shocks versus UV fluorescence}

The near-IR spectrum of $\mathrm{H}_{2}$ can be readily classified as dominated by either thermal collisions or UV-pumped IR fluorescence. The 1-0 S(1)/2-1 S(1) ratio, for example, is characteristically different (10 versus 1.8$)$. In the past, a thermal ratio has generally been taken to imply that the emission is due to shock heated gas and most observations of $\mathrm{H}_{2}$ emission from PNe has been interpreted along these lines (Zuckerman and Gatley 1988). In recent years, it has however been realized that high density PDRs will have characteristics very similar to low velocity shocks; ie., a thin layer of warm molecular gas ( $\$ 2$; Sternberg and Dalgarno 1989; Burton et al. 1990). Thus, they will also give rise to a thermal $\mathrm{H}_{2}$ NIR spectrum. In fact, recent studies of $\mathrm{NGC} \mathrm{7027,} \mathrm{show} \mathrm{that} \mathrm{its} \mathrm{thermal} \mathrm{H}_{2}$ emission occurs from high density PDRs rather than from shocks. First, morphologically, the $\mathrm{H}_{2}$ emission, as well as the $3.3 \mu \mathrm{m}$ PAH emission, is seen to bridge the gap between the ionized gas and 
the CO emission as expected for a PDR (Graham et al. 1992). In this, NGC 7027 resembles well known galactic PDRs such as the Orion Bar (Sellgren et al. 1990). Second, assuming a preshock density of $10^{6} \mathrm{~cm}^{-3}$ (cf., $§ 5$ ), the observed $\mathrm{H}_{2}$ intensity $\left(\approx 10^{-3}\right.$ $\mathrm{erg} /\left(\mathrm{cm}^{2} \mathrm{~s} \mathrm{sr}\right)$ after dereddening) implies a shock velocity of $>10 \mathrm{~km} / \mathrm{s}$ and $>20 \mathrm{~km} / \mathrm{s}$ for a J or C shock, respectively (Burton et al. 1992). However, the $\mathrm{H}_{2}$ line profile reveals a shell expanding at $\approx 15 \mathrm{~km} / \mathrm{s}$ (Fig 3; Geballe 1990). This velocity is very similar to the CO expansion velocity and less than that of HII. Certainly, there is no evidence for shocked gas at $25-35 \mathrm{~km} / \mathrm{s}$ with respect to the stellar systematic velocity required to explain the observed intensity (Graham et al. 1992). Indeed, in general, $\mathrm{H}_{2}$ molecules will only survive inside dense clumps $(\S 3 \& 6)$. Thus, as long as $\mathrm{T}>1000 \mathrm{~K}\left(\mathrm{G}_{\mathrm{o}}>10^{3}\right)$, the $\mathrm{H}_{2}$ spectrum should be thermal. Conversely, a fluorescing $\mathrm{H}_{2}$ spectrum is only expected from the $\mathrm{H}_{2}$ photodissociation wave "racing"through the interclump gas (ie., for young $\mathrm{PN}$ ).

\section{Physical conditions in PDRs around PNe}

Infrared and submillimeter observations can be used in a simple procedure to determine average physical conditions in PDRs. In particular, the ratios of [OI] $63 / 146 \mu \mathrm{m}$ and [OI] $63 /[\mathrm{CII}] 158 \mu \mathrm{m}$ are sensitive to density and temperature. Over much of the relevant parameter space for warm, dense PDRs $\left.\left(10^{2}<\mathrm{n}_{0}<10^{6} \mathrm{~cm}^{-3} ; 100\right)<\mathrm{T}<1000 \mathrm{~K}\right)$, curves of constant ratio of these emission lines are nearly perpendicular in the $\mathrm{n}_{\mathrm{O}}$ - $\mathrm{T}$ plane and thus these ratios give a good indication of the physical conditions in the emission zones (cf., Genzel et al. 1989). Alternatively, rather than using the [OI] $63 \mu \mathrm{m}$ line, which may well be optically thick, one might combine the [OI] 146/[CII] $158 \mu \mathrm{m}$ with the observed heating efficiency. The latter can be determined by comparing the flux in the dominant cooling lines $([\mathrm{OI}]+[\mathrm{CII}])$ with the total IR dust continuum. Then, adopting a dust model, this efficiency can be translated into a relation between $n_{O}$ and $T$ since photoelectric heating efficiency depends on the grain charge which itself depends on these parameters (cf., TH). Although, at first sight, any relation based upon dust physics might be suspect, it should be emphasized that this efficiency relation is semi-empirically founded on a direct observational study of the heating of the ISM (Pottasch et al. 1979). Fortunately, both approaches give very similar results. Moreover, they agree well with other determinations of the physical conditions in PDRs, including such direct ones as the temperature determination from the rotational lines of $\mathrm{H}_{2}$ (Parmar et al. 1992). As a result, such studies have now been applied to a large number of galactic and extragalactic sources (Wolfire et al. 1990; Burton et al. 1990).

Table 1 summarizes the results obtained this way for the PDR in NGC 7027 and compares them to those for the Orion Bar, the galactic center and M82. We conclude from this comparison that the physical conditions in these PDRs are all very similar, with average densities of $\approx 10^{5} \mathrm{~cm}^{-3}$ and temperatures of $\approx 500 \mathrm{~K}$. However, the observations also imply the existence of higher density clumps $\left(10^{6}-10^{7} \mathrm{~cm}^{-3}\right)$ with a small volume filling fraction (Burton et al. 1990). We suggest that, in NGC 7027, this dense gas is part of the highly clumped circumstellar torus (diameter $\approx 6 ")$, obvious in $\mathrm{Br} \alpha, \mathrm{H}_{2}$ 1-0 S(1), and $3.3 \mu \mathrm{m}$ feature (Graham et al. 1992). With an expected scale size of $\approx 5 \times 10^{15} \mathrm{~cm}\left(0.3^{\prime \prime}\right)$, the PDR will form an unresolved layer outside the ionized gas in this torus. The expected HI column density for such high density gas is small $\left(10^{20} \mathrm{~cm}^{-2}\right.$; cf., §2). Coupled with its high temperature $(\approx 2000)$, this may explain the non-detection of HI absorption in NGC 
Table 1: Physical conditions in PDRs derived from atomic finestructure lines.

\begin{tabular}{cccccc} 
Object & NGC 7027 & Orion Bar & Sgr A & M82 & notes \\
\hline $\mathrm{n}\left(\mathrm{cm}^{-3}\right)$ & $7 .(4)$ & $1 .(5)$ & $1 .(5)$ & $5 .(4)$ & 1 \\
$\mathrm{~T}(\mathrm{~K})$ & 400 & 500 & 750 & 400 & 2 \\
$\mathrm{G}_{\mathrm{O}}$ & $1 .(4)$ & $4 .(4)$ & $1 .(5)$ & $1 .(4)$ & 3 \\
$\mathrm{M}_{\mathrm{a}} / \mathrm{M}_{\mathrm{m}}$ & 0.1 & 0.04 & 0.04 & 0.1 & 4 \\
$\mathrm{n}_{\mathrm{c}}$ & $1 .(6)$ & $1 .(7)$ & $1 .(7)$ & - & 5 \\
$\mathrm{f}_{\mathrm{v}}$ & 0.3 & 0.1 & 0.05 & - & 6
\end{tabular}

notes: 1) Average density. 2) Average Temperature. 3) Incident UV field in units of the average interstellar radiation field $\left(1.6 \times 10^{-3} \mathrm{erg} \mathrm{cm}^{-2} \mathrm{~s}^{-1}\right.$; Habing 1968). 4) Ratio of atomic to molecular gas mass. 5) Clump density. 6) Clump volume filling factor. Ref. Wolfire et al. (1990) \& Burton et al. 1990).

7027 (Huggins 1992). Most of the material in these clumps $\left(\mathrm{N} \approx 2 \times 10^{22} \mathrm{~cm}^{-2}\right)$ is in molecular form. The lower density PDR gas evident from the atomic finestructure lines is likely associated with the low surface brightness (fluorescing) $\mathrm{H}_{2}$ and PAH emission extending about 3 " further out from the ionized gas (Graham et al. 1992). The density (10 5 $\mathrm{cm}^{-3}$ ) derived from the observed scale size of the emission is in good agreement with that derived from the FIR lines (Table 1). The absence of $\mathrm{HI}$ absorption of this gas $\left(\mathrm{N}_{\mathrm{H}}=4 \times 10^{21} \mathrm{~cm}^{-2}\right)$ against the HII region suggests that it is located in two lobes, above and below the ionized torus.

\section{Clumps}

A clumped distribution of molecular emission seems to be an inherent property of all PNe (Huggins 1992; Cox et al. 1992). As discussed in $\$ 3$, high density clumps are a necessity for molecular survival and/or an active chemistry in PN ejecta. These clumps may reflect instabilities during the superwind phase when the torus was created or result from Rayleigh-Taylor instabilities during the PN phase when the hot bubble pressed upon the superwind (cf., Balick elsewhere in this volume). The pressure in the NGC 7027 neutral clumps, $\approx 10^{9} \mathrm{~cm}^{-3} \mathrm{~K}$, is comparable to the pressure of the ionized gas $\left(\mathrm{n}_{\mathrm{c}}=6 \times 10^{4}\right.$ $\mathrm{cm}^{-3}, \mathrm{~T}_{\mathrm{e}}=14,500 \mathrm{~K}$; Roelfsema et al. 1991). During the ionization phase of the PN, the pressure on the clumps will be constant (Marten and Schönberner 1991). Since the incident FUV field $(\leq 13.6 \mathrm{eV})$ will decrease due to geometrical dilution, the temperature in the neutral gas will slowly drop and the density increase in this phase. During the expansion phase $\left(t_{\mathrm{exp}}>3000 \mathrm{yr}\right)$, the pressure on the clump will decrease and the clump density will start to decrease.

As for neutral globules in HII regions, the lifetime of these clumps will be determined by erosion due to photo-ionization (Huggins et al. 1992). The stellar ionizing photon flux, $N_{*}$, will be attenuated by the ionized layer on the clump surface. The flux of photons, $N$, arriving at the ionization front of a clump of size $\mathrm{R}_{\mathrm{c}}$ at a distance $\mathrm{r}$ from the star is then approximately given by $\left.N \approx\left\{\left[3 N * / 4 \pi \mathrm{r}^{2}\right] \mathrm{C}^{2} / \alpha \mathrm{R}_{\mathrm{c}}\right]\right\}^{0.5}$ with $\alpha$ the recombination coefficient and $\mathrm{C}$ the sound speed in the ionized gas (Spitzer 1978). Adopting a hot black body, $N * \approx 6.3 \times 10^{52} / \mathrm{T}_{\mathrm{eff}}$ photons $\mathrm{s}^{-1}$ for $\mathrm{L}=6000 \mathrm{~L}_{\odot}$ and $N \approx 2 \times 10^{11}$ $\left[10^{5} \mathrm{~K} / \mathrm{T}_{\mathrm{eff}}\right]^{0.5}\left[5 \times 10^{16} / \mathrm{r}(\mathrm{cm})\right]$ photons $\mathrm{s}^{-1}$. Thus, the column density eroded by 
photoionization is then given by $\mathrm{N}(\mathrm{H})=\int N \mathrm{dt}$, which is only weakly dependent on time for a clump coasting at a constant velocity; ie., $\mathrm{N}(\mathrm{H}) \approx 7 \times 10^{21}\left[10^{5} \mathrm{~K} / \mathrm{T}_{\mathrm{eff}}\right]^{0.5}[15 / \mathrm{v}(\mathrm{km} / \mathrm{s})]$

$\ln \left[\mathrm{t}_{\mathrm{exp}} / \mathrm{t}_{0}\right] \mathrm{cm}^{-2}$ where $\mathrm{t}_{\mathrm{o}}$ is the time at which the ionizing flux is turned on $(\approx 500 \mathrm{yr})$. Thus, clumps with column densities of at least $2 \times 10^{22} \mathrm{~cm}^{-2}$ will survive throughout the PN phase. Such clumps are optically thick in the UV and thus molecules will survive.

Moreover, adopting a typical clump scale size, $R_{c} \approx r_{j} / 3 \approx 10^{15}-10^{16} \mathrm{~cm}$ with $r_{i}$ the shell size at clump formation, the density is high enough to drive an active chemistry. In conclusion, clumps that survive during the PNe phase are by necessity molecular.

ACKNOWLEDGEMENTS: This review benefitted greatly from stimulating discussion with and preprints made available by Pierre Cox, James Graham, David Hollenbach, and Pat Huggins. Theoretical studies of photodissociation regions and their observational characteristics at NASA Ames are supported through NASA grant 188-41-53.

\section{REFERENCES}

Burton, M.G., Hollenbach, D.J., \& Tielens, A.G.G.M., 1990, ApJ, 365, 620.

Burton, M.G., Hollenbach, D.J., \& Tielens, A.G.G.M., 1992, ApJ, Nov, in press.

Cox, P., Omont, A., Huggins, P.J., Bachiller, R., and Forveille, T., AA, in press.

Dinerstein, H.L., 1991, PASP, 103, 861.

Geballe, T.R., 1990, in Molecular Astrophysics, ed. T. Hartquist, (Cambridge Univ Press, Cambridge), p.345.

Genzel, R., Harris, A.I. \& Stutski, J., 1999, in IR Spectroscopy and Astronomy, ed.

Kaldeich, B.H., ESA, SP-290, p115.

Graham, J.R., et al., 1992, ApJ, in press.

Habing, H.J., 1968, Bull. Astr. Inst. Neth., 19, 421.

Hill, J.K., \& Hollenbach, D.J., 1978, ApJ, 225, 390.

Hollenbach, D.J., Takahashi, T., \& Tielens, A.G.G.M, 1991, ApJ, 377, 192.

Huggins, P.J., and A.P. Healy, 1989, ApJ, 346, 201.

Huggins, P.J., 1992, in Mass Loss on the AGB and Beyond, ESO, in press.

Huggins, P.J., Bachiller, R., Cox, P., and Forveille, T., 1992, ApJL, Sept, in press.

Jaminet, P.A., et al., ApJ., 380, 461.

Marten, H. \& Schönberner, D., 1991, A\&A, 248, 590.

Parmar, P.S., Lacy, J. \& Achtermann, J.,1992, ApJL, 372, L25.

Pottasch, S.R., 1980, A\&A, 89,336.

Roelfsema, P.R., Goss, W.M., Pottasch, S.R., and Zijlstra, A., 1991, A\&A, 251, 611.

Sopka, R.J., et al, 1985, ApJ, 294, 242.

Spaans, M., van Dishoeck, E.F., \& Tielens, A.G.G.M., 1992 in preparation.

Spitzer, L., 1978, Physical Processes in the Interstellar Medium, (Wiley and Sons, NY).

Sternberg, A. \& Dalgarno, A., 1989, ApJ, 338, 197.

Taylor, A.R., Gussie, G.T. \& Pottasch, S.R., 1990, Ap.J., 351, 515.

Tielens, A.G.G.M. \& Hollenbach, D.J., 1985, ApJ, 291, 722 (TH).

Tielens, A.G.G.M. and Allamandola, L.J., 1987, in Interstellar Processes, eds. D.

Hollenbach and H.Thronson, (Reidel, Dordrecht), p.397.

van Dishoeck, E.F. \& Black, J.H., 1988, ApJ, 334, 771.

Wolfire, M.G., Tielens, A.G.G.M, \& Hollenbach, D.J., 1990, ApJ, 358, 116.

Zuckerman, B. \& Gatley, I., 1988, Ap.J, 324, 501. 